\title{
Effect of System Parameters on Phase Error of Focus-Free Digital Fringe Projection Profilometry System
}

\author{
H. A. Ali ${ }^{1}$, M. A. Amer ${ }^{1}$, A. A. Omara ${ }^{2}$. \\ ${ }^{1}$ Engineering and surface metrology lab., Length and pression engineering division, National \\ Institute for Standards (NIS), Egypt. \\ ${ }^{2}$ physics department, faculty of science, Al-Azhar university (girls branch), Egypt.
}

\begin{abstract}
Digital Fringe projection profilometry (DFPP) is a noncontact, fast, accurate and flexible technique for 3D measurements. Phase calculation is an important step that affects the accuracy of measurements. in phase calculation, the critical source of error is phase error due to nonlinear gamma effect of the system. This paper introduces an experimental investigation of the nonlinear gamma effect of DFPP system as well as the effect of system parameters, such as fringe intensity, fringe period, surface distance from the camera and projector and finally the ambient illumination, on phase error and consequently system measurements. Fringe patterns are generated using Matlab 2018a and projected by digital laser projector. The deformed patterns are captured using colored CCD camera. Phase shifting technique is used to calculate phase map. The system shows a linear response in the intensity range (50-220) and has small phase error without any compensation for nonlinear gamma effect. The phase error of the system slightly increases with increasing fringe period and the surface distance from the camera and projector, while it is nearly constant with ambient illumination changes from dark to median level and shows a bigger change if the ambient is completely illuminated. In the worst conditions the phase error does not accedes 0.0387 rad without any compensation for nonlinear gamma effect, make the system a useful tool for 3D measurements in different working fields.
\end{abstract}

Keywords: Digital Fringe Projection Profilometry (DFPP), Phase error, Focus-free laser projector.

Receive Date: 17 September 2021; Revise Date: 3 October ; Accept Date: 05 October 2021.

Publish Date: 08 October

Corresponding Author Email: hanem_nis@yahoo.com 


\section{1- Introduction}

Three-dimensional (3D) measurements have various applications in different fields such as biomedical engineering, machine vision, medical practice, reserve engineering, quality assurance, biometric security, and entertainment ${ }^{[1-3]}$. 3D shape could be retrieved using contact techniques such as Coordinate Measuring Machine (CMM). These techniques are limited by slow performance, obligation of touching the measured object and high cost. Non-contact techniques such as time-of-flight (TOF) method, interferometry method, Digital Fringe projection profilometry (DFPP), and laser scanning method have the advantage of overcoming the previous limitations ${ }^{[3,4]}$. DFPP is one of the most used noncontact techniques for 3D imaging ${ }^{[5]}$ due to its speed, accuracy, and flexibility ${ }^{[6-9]}$.

DFPP system mainly consists of digital projector and CCD camera which are placed at a distance away from a measured object with certain geometrical design. Digital projector is used to project uniform fringe patterns, generated by MATLAB 2018a, on the object surface. These patterns are deformed due to the object height distribution. Image of the deformed patterns is captured by CCD camera ${ }^{[10,11]}$ then analyzed by one of fringe analysis techniques such as Fourier transform ${ }^{[12]}$, wavelet transform ${ }^{[13]}$, and phase shifting technique ${ }^{[14]}$ to obtain phase map, then unwrapped to get continuous phase map. Finally, a calibration step is done to transform from continuous phase map to $\mathrm{X}, \mathrm{Y}, \mathrm{Z}$ coordinates ${ }^{[15,16]}$.

Performance of DFPP system is characterized by many factors such as resolution, speed and the most important factor is accuracy. The accuracy is affected by several factors such as system components, system calibration, geometric and surface characteristics of tested object, system set up and ambient illumination ${ }^{[3]}$.

The height of the object (h) could be related to phase shift caused by the object by the following relation

$$
h=\frac{\Delta \varphi \mathrm{L}}{2 \pi \mathrm{fD}+\Delta \varphi}
$$

where :-

$\mathrm{L}$ is the distance between the reference plane and the camera $(\mathrm{C})$ and projector $(\mathrm{P})$.

$\mathrm{D}$ is the distance between the optical axis of the camera and projector.

$F$ is the frequency of fringe patterns

$\Delta \varphi$ is the phase shift value caused by the object height ${ }^{[16]}$, these parameters are shown schematically in figure (2)

Since $\mathrm{L}$ and $\mathrm{D}$, and $\mathrm{f}$ are fixed values for the system, the accuracy of height measurements depends on the accuracy of calculating the phase shift caused by the object height.

It is known that projector and CCD camera do not response linearly to input intensities, 
this behavior is known as nonlinear gamma response. Therefore, when sinusoidal fringes are used, they are not projected or captured with the same generated ideal waveform causing a phase error when phase is retrieved ${ }^{[17]}$. Determination of phase shift is mainly affected by phase error due to nonlinear gamma effect. Many researchers attempted to compensate phase error using active methods by modifying the fringe intensities before projecting ${ }^{[18,19]}$ or by passive methods after capturing fringe images and correcting the phase map ${ }^{[20-21]}$. In spite of the many attempts for compensating phase error, there is a lacking in researches investigating the relation between system parameters i.e., fringe intensity, fringe period, surface distance from the projector and camera and Ambient illumination, and phase error.

In this study a new type of projector, laser projector, is used to project digital fringes. Laser projector has the advantage of focus-free projection. It does not need any special type of lenes to control the depth of field, but it already projects focused images at distance up $1500 \mathrm{~mm}$ without any additional lenses or manual adjusting. This work introduces experimental investigation of the effect of system parameters (fringe intensity, fringe period and surface distance from projector and camera) and ambient illumination level on the phase error. The rest of this paper is organized as following: section 2 presents the principle of three steps phase-shifting algorithm to calculate the phase map. Section 3 explains the experimental work and results, and Section 4 gives the conclusion of this work.

\section{2- $\quad$ Three steps phase-shifting algorithm}

Fringe analysis is an important step that control the performance of any DFP system. Among fringe analysis techniques phase shifting profilometry (PSP) has the advantages of being retrieve accurate phase, can achieve pixel-wise phase measurement with high resolution and robust to ambient illumination and varying surface reflectivity, so (PSP) found its way to be one of the most widely used fringe analysis techniques ${ }^{[22]}$. Many different algorithms were developed such as standard N-step phase shifting algorithm, double three-step phase shifting algorithm, trapezoidal phase shifting, triangular phase shifting, and Hariharan 5-step phase shifting ${ }^{[23]}$. In general cases, at least three phase shifted fringe patterns image are needed when standard N-step phase shifting algorithm is used to calculate phase map. Intensity of the three waves shifted by $2 \pi / 3 \mathrm{rad}$ are $\mathrm{I}_{1}, \mathrm{I}_{2}, \mathrm{I}_{3}$ as shown in figure (1) represented by the following equations

$$
\begin{aligned}
& \mathrm{I}_{1}(\mathrm{x}, \mathrm{y})=\mathrm{I}^{\prime}(\mathrm{x}, \mathrm{y})+\mathrm{I}^{\prime \prime}(\mathrm{x}, \mathrm{y}) \cos \{\varphi(\mathrm{x}, \mathrm{y})-2 \pi / 3\} \\
& \mathrm{I}_{2}(\mathrm{x}, \mathrm{y})=\mathrm{I}^{\prime}(\mathrm{x}, \mathrm{y})+\mathrm{I}^{\prime \prime}(\mathrm{x}, \mathrm{y}) \cos \{\varphi(\mathrm{x}, \mathrm{y})\} \\
& \mathrm{I}_{3}(\mathrm{x}, \mathrm{y})=\mathrm{I}^{\prime}(\mathrm{x}, \mathrm{y})+\mathrm{I}^{\prime \prime}(\mathrm{x}, \mathrm{y}) \cos \{\varphi(\mathrm{x}, \mathrm{y})+2 \pi / 3\}
\end{aligned}
$$


Where $I^{\prime}(x, y)$ is the average intensity, $I^{\prime \prime}(x, y)$ is the intensity modulation and $\varphi(x, y)$ is the phase. By solving the three equations the extracted phase will be

$$
\varphi(\mathrm{x}, \mathrm{y})=\tan ^{-1}\left[\frac{\sqrt{3}\left(I_{1}-I_{3}\right)}{\left(2 I_{2}-I_{1}-I_{3}\right)}\right]
$$

The calculated phase values are ranging from $-\pi$ to $\pi$, so a suitable phase unwrapping algorithm is used to remove $2 \pi$ discontinuities.

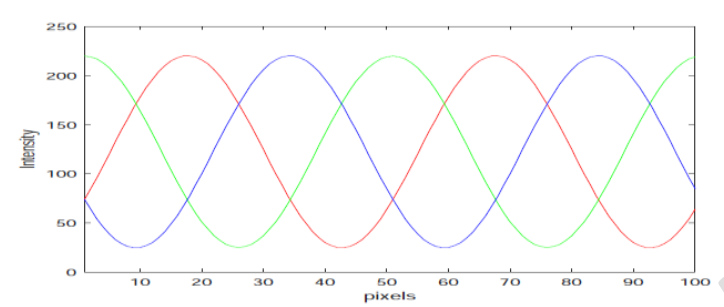

Figure (1). Three sinusoidal waves shifted by $2 \pi / 3$ rad. $I_{1}$ in red color, $I_{2}$ in green color, and $I 3$ in blue color.

\section{3- Experimental work and Results.}

In this work a colored CCD camera Model EO-18112 manufactured by Edmund optics, a lens model FL\#33-302 manufactured by Edmund optics, focus-free laser mobile pico projector Model MP-CL1A manufactured by Sony. This device emits class 3R laser with wavelength from $445 \mathrm{~nm}$ to $639 \mathrm{~nm}$ and beam divergence $4.5 \mathrm{mrad}$, and a computer manufactured by Dell, model latitude 5580 are used to perform the experimental work of this paper. The system setup is that the optical axis of the camera and the projector are parallel to each other as shown in the schematic diagram of the constructed fringe projection profilometry system illustrated in figure (2).

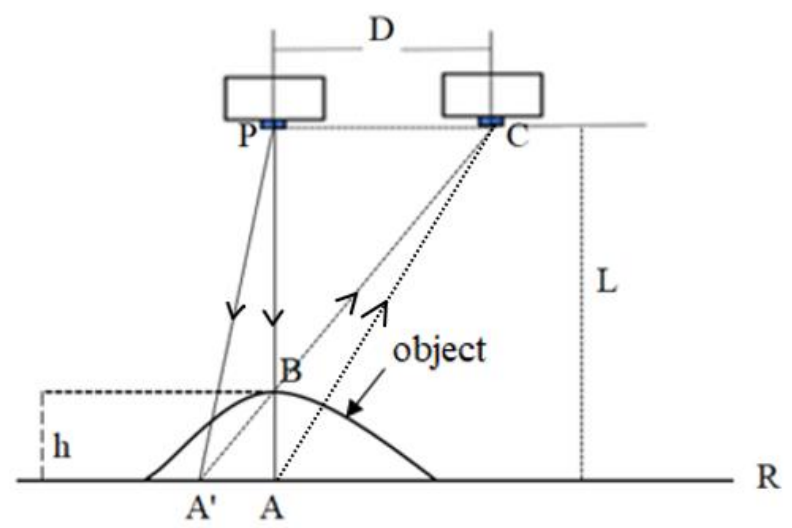

Figure (2). Schematic diagram of fringe projection profilometry system

\section{3-1 Nonlinear gamma effect}

Nonlinear gamma response of the system was studied by projecting a set of green images generated with different intensities between 20 and 220 with fixed step value 10 on a 
reference surface using the laser projector. This set of generated intensities is considered as input intensity $\left(\mathrm{I}_{\mathrm{o}}\right)$. These projected images are imaged by the colored CCD camera, then green channel of the image is separated to be used in the analysis. Average value of a small area $(10 \times 10)$ pixels in the center of the captured images is calculated and considered as the output intensity (I). Figure (3) shows nonlinear gamma effect curve. The relation between $\left(\mathrm{I}_{\mathrm{o}}\right)$ and $(\mathrm{I})$ is represented by a polynomial of third order.

$$
\mathrm{I}=(-1.5903) \times 10^{-5}\left(\mathrm{I}_{\mathrm{o}}\right)^{3}+0.00718\left(\mathrm{I}_{\mathrm{o}}\right)^{2}+0.14429\left(\mathrm{I}_{\mathrm{o}}\right)-2.777
$$

In the following section effect of fringe intensity range on phase error value will be studied.

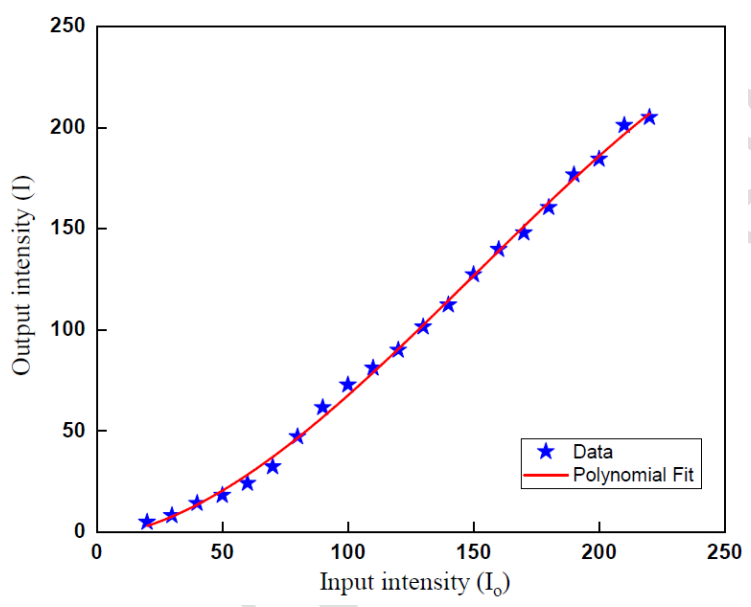

Figure (3). Nonlinear gamma effect curve. polynomial fitting for intensity range (20-220).

\section{3-1 Effect of fringe intensity}

Effect of fringe intensity on phase error is investigated at different intensity amplitudes [ (25-150), (25-175), (25-200), (25-220), (50-150), (50-175), (50-200) and (50-220)] using three sinusoidal fringe images shifted by $2 \pi / 3$ of each intensity amplitude projected on a reference surface as shown in figure (4). Fringe images were captured by the camera then, phase was calculated using equation (5) and unwrapped to remove $2 \pi$ discontinuities Unwrapping was done using a free Matlab code based on the reference ${ }^{[24]}$. Unwrapped phase takes the range between 0 and $n \pi$ and the relation between unwrapped phase and pixels coordination is linear. For better visualization of phase error, a linear fitting, for the pixels- unwrapped phase relation, is calculated and subtracted from the measured unwrapped phase values then the final phase error is calculated by obtaining Root Mean Square (RMS) of the resulting data. Figure (5) shows phase error at $500^{\text {th }}$ row of the unwrapped phase map associated with each intensity range ${ }^{[25]}$. RMS values of phase error at each intensity are listed in table (1). Figure (6) shows that when intensity lower limit is 25 , the (RMS) value of phase error decreases from 0.0412 to $0.0243 \mathrm{rad}$ as the upper limit 
of fringe amplitude increases. At the same time, when lower limit value is raised to 50 the phase error is nearly constant with small RMS value does not accede 0.0234 rad whatever the upper limit is without any compensation for nonlinear gamma effect. This behavior indicates the more linearity of the system in intensity range (50-220) where the data could be fitted linearly as shown in figure (7).

$$
\mathrm{I}=1.14275 \times\left(\mathrm{I}_{\mathrm{o}}\right)-44.0850
$$

Since the intensity amplitude (50-220) has the lowest phase error, this range is used in the rest work of this paper.

\begin{tabular}{|c|c|}
\hline Intensity range & (RMS) Phase error (rad) \\
\hline $25-150$ & 0.0412 \\
$25-175$ & 0.0350 \\
$25-200$ & 0.0335 \\
$25-220$ & 0.0243 \\
$50-150$ & 0.0227 \\
$50-175$ & 0.0220 \\
$50-200$ & 0.0234 \\
$50-220$ & 0.0220 \\
\hline
\end{tabular}

Table (1). RMS values of Phase error at different intensity ranges

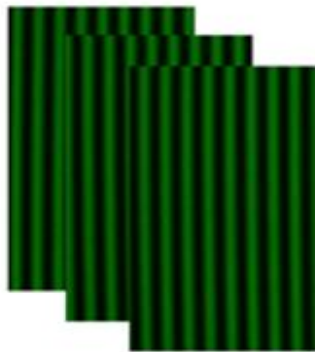

(a)

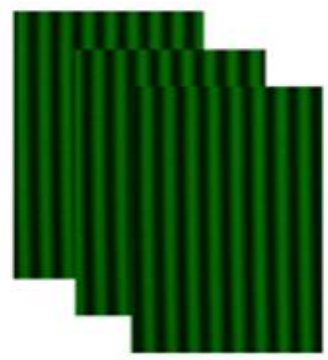

(d)

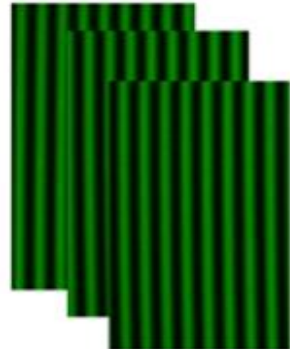

(b)

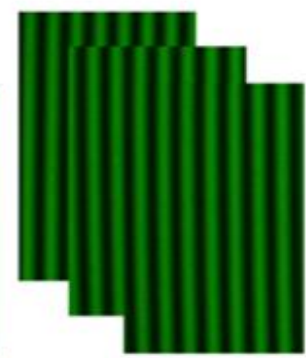

(e)

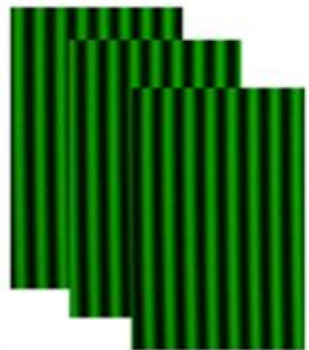

(c)

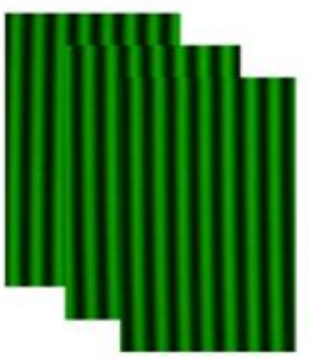

(f)

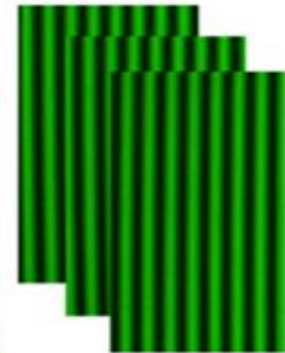

(d)

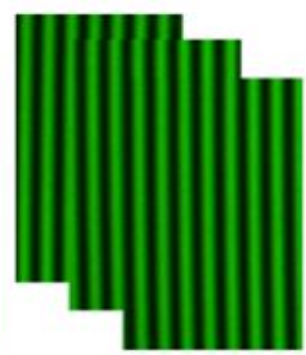

(g)

Figure (4). Captured fringe patterns images of different intensities. (25-150) (a), (25-175) (b), (25-200) (c), (25-220) (d), (50-150) (e), (50-175) (f), (50-200) (g), and (50-220) (h). 
(a)
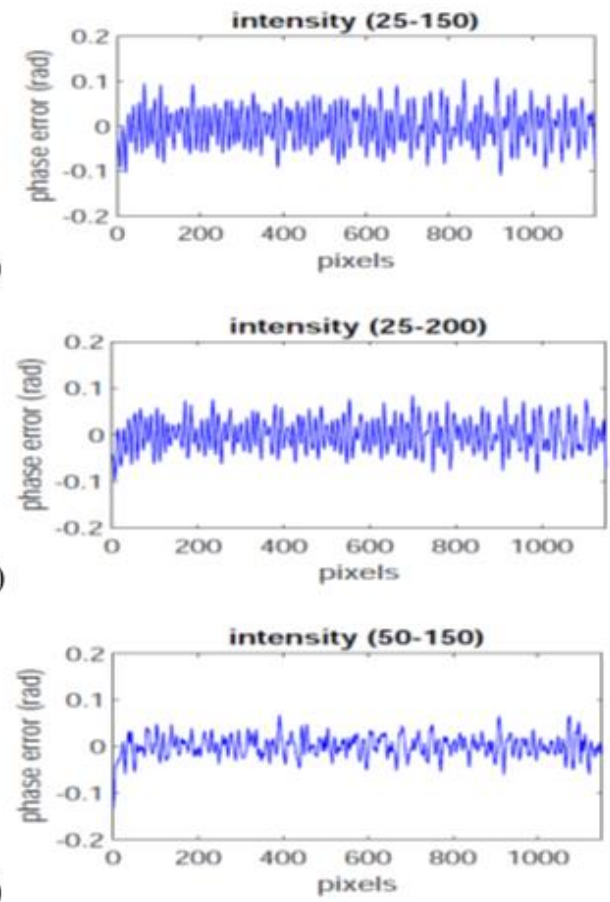

(e)

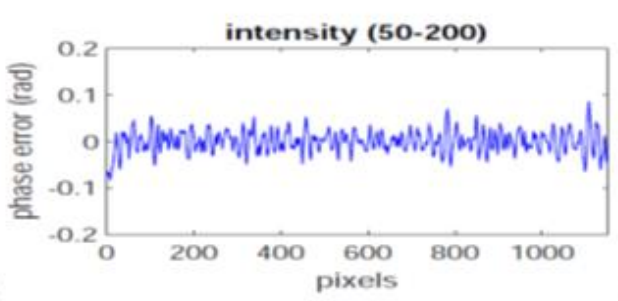

(b)

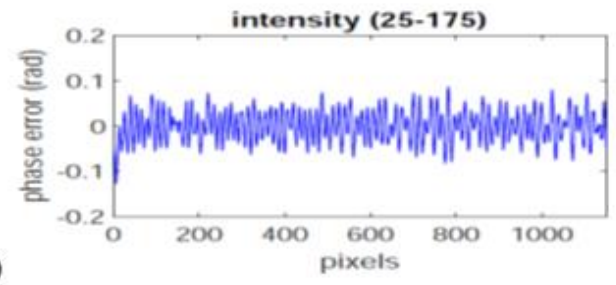

(d)
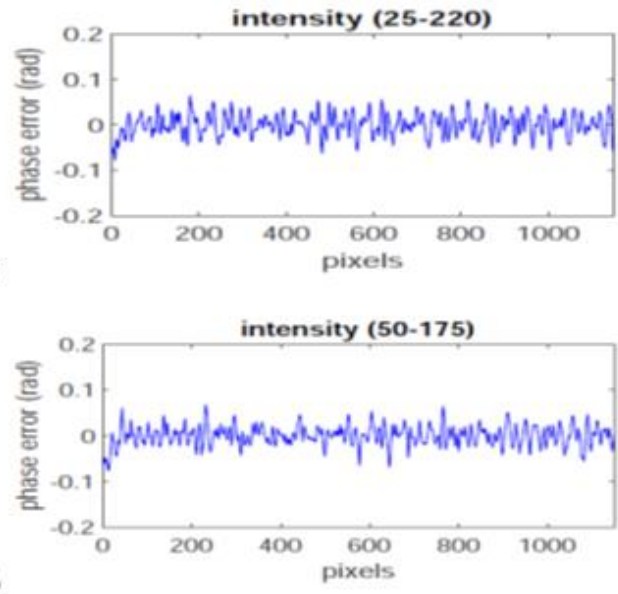

(f)

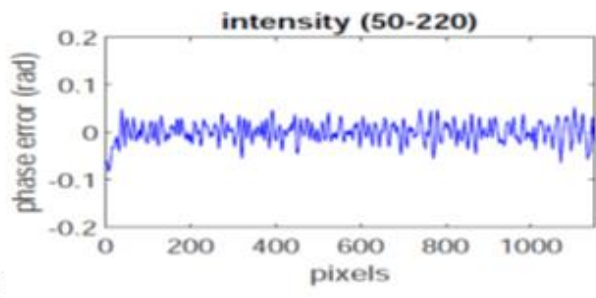

Figure (5). Phase error for intensity ranges. (25-150) (a), (25-175) (b), (25-200) (c), (25-220) (d), (50-150) (e), (50-175) (f), (50-200) (g), and (50-220) (h).

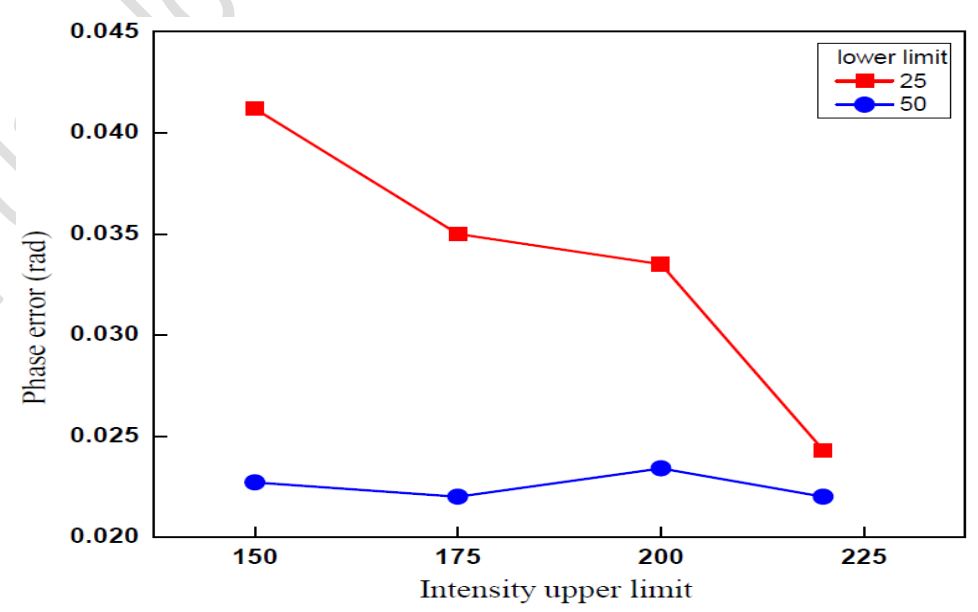

Figure (6). Change of phase error of $500^{\text {th }}$ row of phase map at different intensity ranges. 


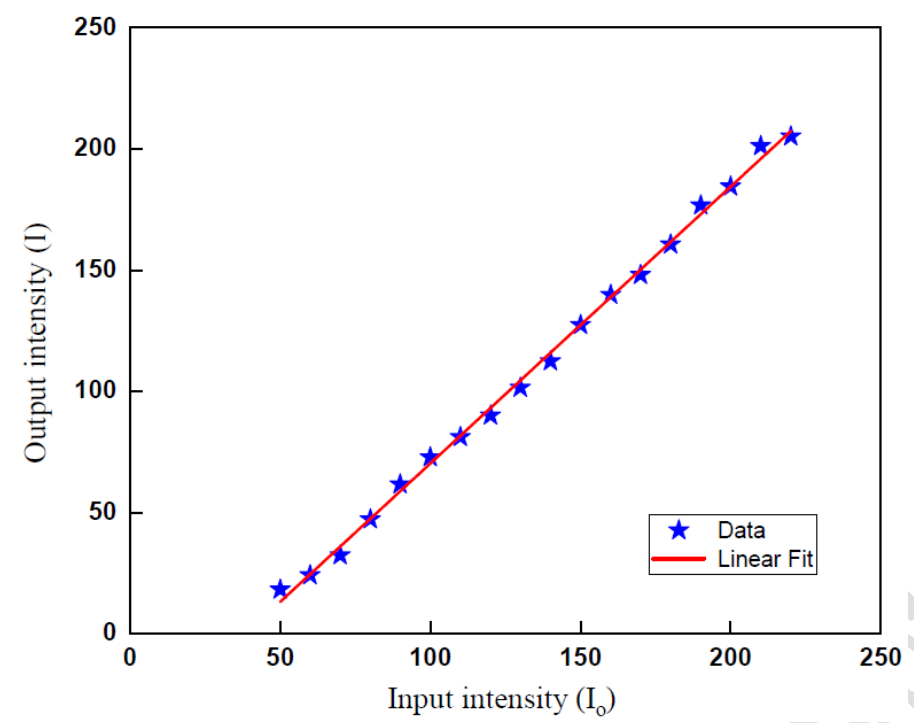

Figure (7). Linear fitting of nonlinear gamma effect curve for intensity range (50-220).

\section{3-2 Effect of fringe period.}

To investigate how the fringe period affects the phase error, three sinusoidal fringe images shifted by $2 \pi / 3$ and with intensity amplitude (50-220) and different fringe periods $(25,30,35$ and 40 pixels) are projected on a reference surface. Fringe images were captured by the camera as shown in figure (8) then, phase was calculated using equation (5) and unwrapped to remove $2 \pi$ discontinuities. Figure (9) shows cross section of $500^{\text {th }}$ row of the unwrapped phase map after removing slop for better showing phase error associated with each fringe period. Phase error is calculated accordingly, and RMS values are listed in table (2). When sinusoidal fringe patterns are used, each pixel will have a unique intensity value. If fringe period $(2 \pi)$ is represented by little number of pixels, the difference between the intensity of the adjacent pixels is large to some extent that the camera and projector sensors can easily differentiate, so the captured sinusoidal fringe image has a small deformation. Consequently, the phase error associated with small fringe periods is small. As the fringe period increases, the difference in intensity values of the adjacent pixels is reduced to some extent that the camera and projector sensors can't differentiate correctly between them, cause the deformation of the captured sinusoidal fringes. As a result, the phase error increases by increasing the fringe period. This appears as increasing RMS value of phase error from $0.0228 \mathrm{rad}$ at fringe period 25 pixels to $0.0257 \mathrm{rad}$ at fringe period 40 pixels. 


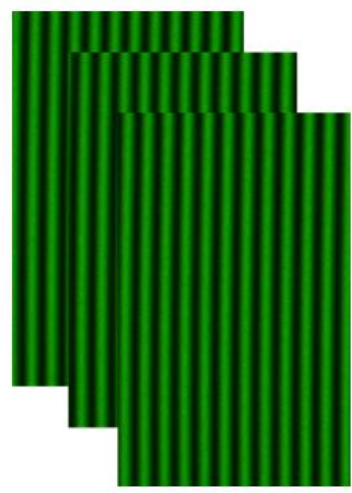

(a)

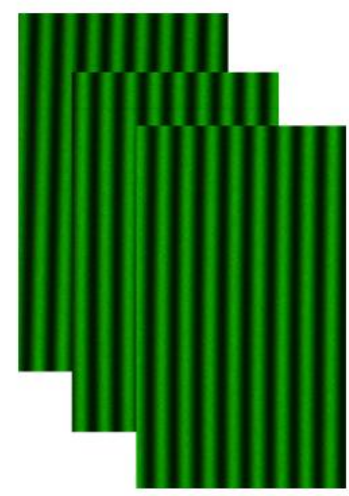

(b)

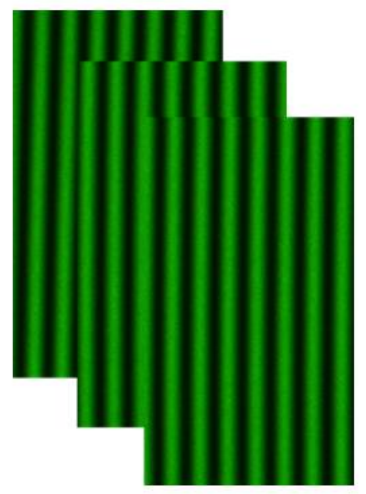

(c)

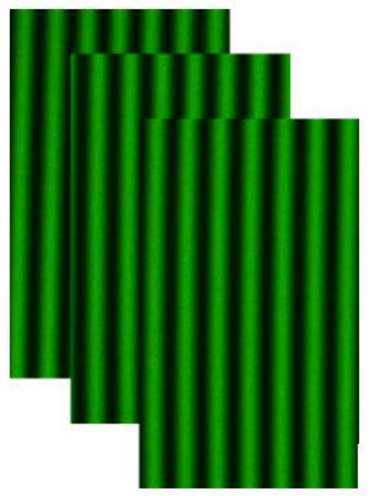

(d)

Figure (8). Captured fringe patterns images of different fringe periods. (a) 25 pixels, (b) 30 pixels, (c) 35 pixels, and (d) 40 pixels.

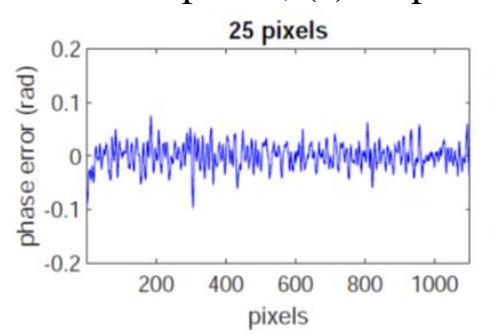

(a)

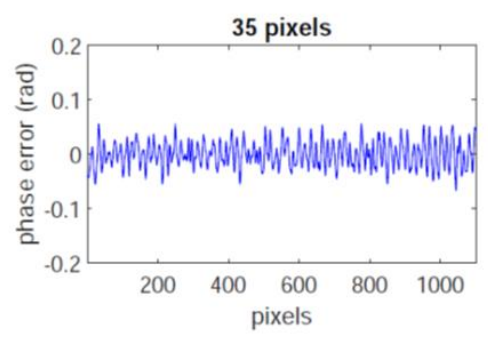

(c)

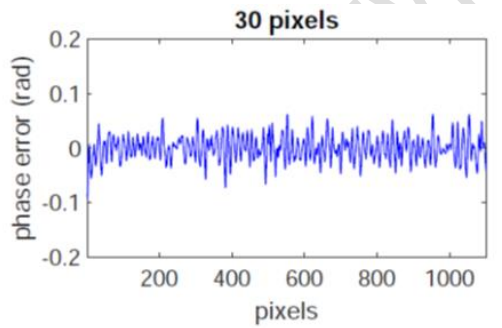

(b)

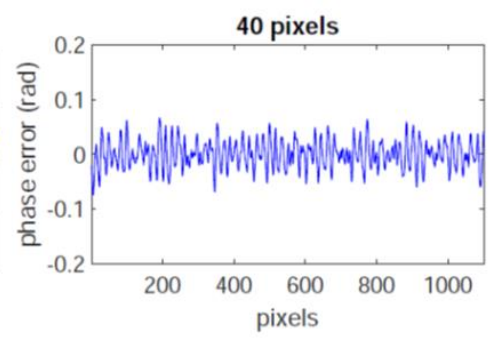

(d)

Figure (9). Phase error of $500^{\text {th }}$ row for different fringe periods. 25 pixels (a), 30 pixels (b), 35 pixels (c) and 40 pixels (d).

To show the effect of fringe period on phase error and system measurements, a cylindrical object is tested using fringe images with different periods $(25,30,35$ and 40 pixels). Three sinusoidal fringe images shifted by $2 \pi / 3$ of each fringe period are projected on a reference surface placed at $500 \mathrm{~mm}$. images of projected fringes are captured by the camera and phase map is calculated using equation (5) and unwrapped at each fringe period. Again, the unwrapped phase maps are calculated with the presence of the object. Figure (10) shows the captured images of different fringe periods projected on the cylindrical object. The phase shift due to the object existence is calculated by subtracting the object unwrapped phase map and the reference unwrapped phase map at each fringe 
period and listed in table (2). If the object surface is placed at certain distance, the lateral shift due to the object height is constant and represented by fixed number of pixels. At the same time, this number of pixels represent a different values of phase shift when fringe patterns with different fringe period is used. the value of phase shift due to the object height decreases as the fringe period increases as shown in figure (11). Because of increasing the phase error and decreasing phase shift with increasing fringe period, the error in phase calculation as a percentage of the measured value will increase from $0.236 \%$ at fringe period 25 pixels to $0.425 \%$ at fringe period 40 pixels as shown in table (2). so, it is preferred to use fringe period with small number of pixels to get more accurate measurements.

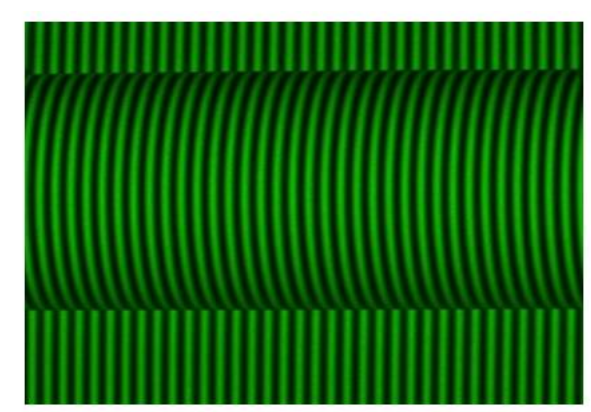

(a)

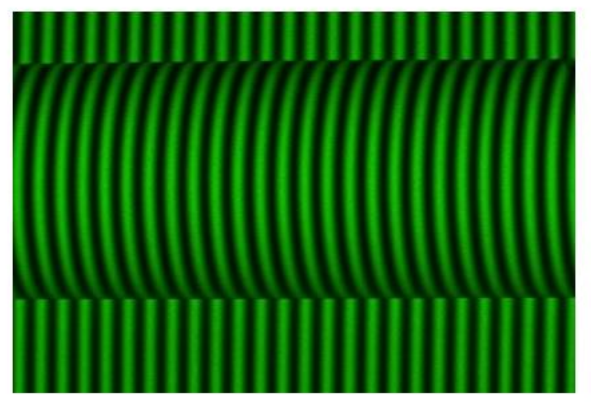

(c)

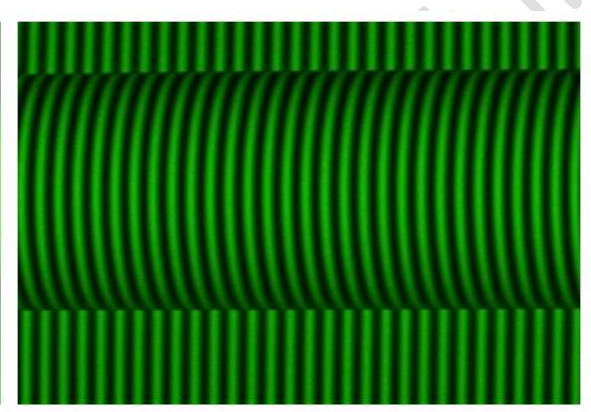

(b)

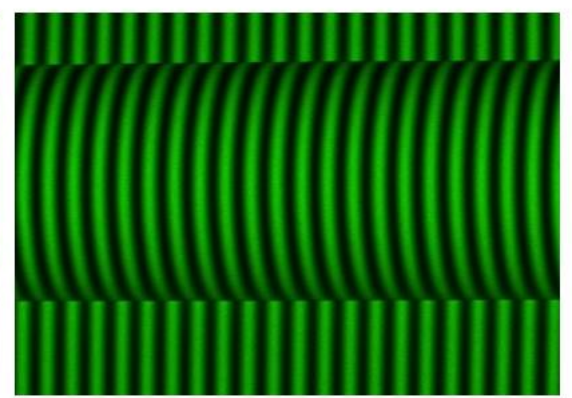

(d)

Figure (10). Captured images of different fringe period projected on test object. 25 pixels (a), 30pixels (b), 35 pixels (c), and 40 pixels (d).

\begin{tabular}{|c|c|c|c|}
\hline $\begin{array}{c}\text { Fringe period } \\
\text { Pixels }\end{array}$ & $\begin{array}{c}\text { Phase error (RMS) } \\
\text { Rad }\end{array}$ & $\begin{array}{c}\text { Phase shift } \\
\text { rad }\end{array}$ & $\begin{array}{c}\text { Error } \\
\mathbf{\%}\end{array}$ \\
\hline 25 & 0.0228 & 9.6661 & 0.236 \\
30 & 0.0225 & 8.0551 & 0.279 \\
35 & 0.0238 & 6.8848 & 0.346 \\
40 & 0.0257 & 6.0478 & 0.425 \\
\hline
\end{tabular}

Table (2). Phase error and phase shift at different fringe period. 


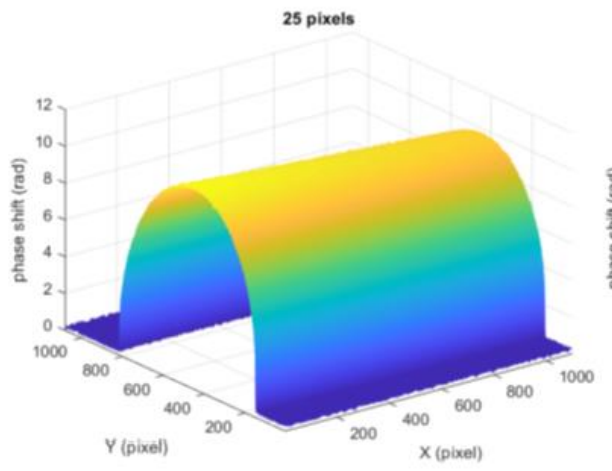

(a)

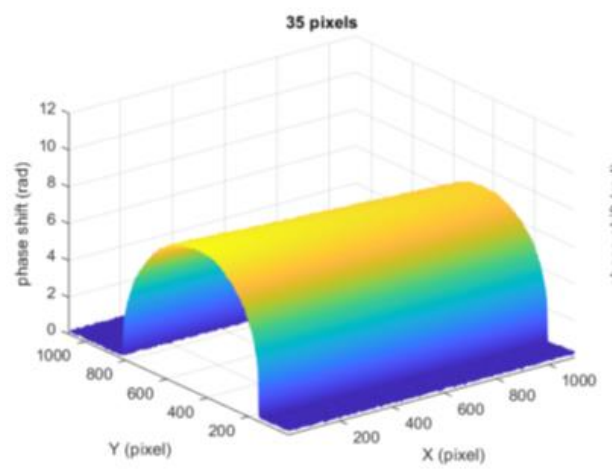

(c)

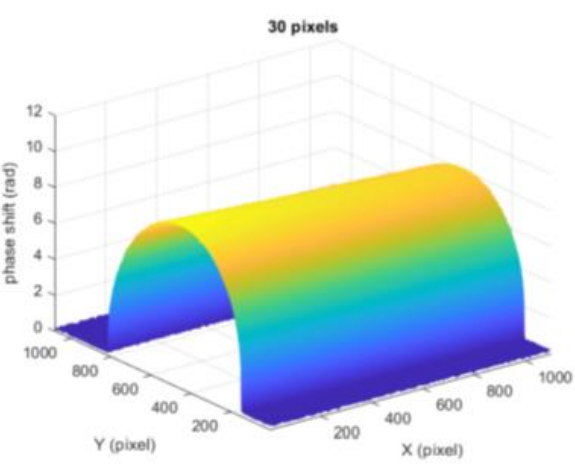

(b)

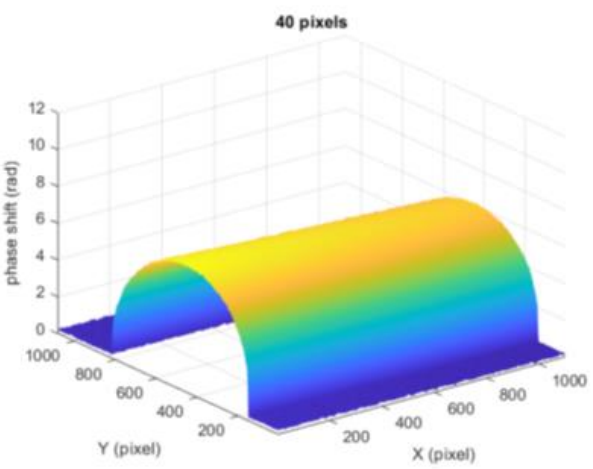

(d)

Figure (11). Phase shift maps for different fringe periods. 25 pixels (a), 30 pixels (b), 35 pixels (c), and 40 pixels (d).

\section{3-3 Surface distance to the camera and projector.}

One of the geometrical parameters of the fringe projection profilometry system is the distance between the object surface and the system (camera \& projector). This parameter is tested at 500, 1000, and $1500 \mathrm{~mm}$. Three sinusoidal fringe images shifted by $2 \pi / 3$, fringe period equals to 35 pixels and intensity amplitude (50-220) are projected on a reference surface at each distance.The camera parameters such as (lens aperture, gain, exposure time) need to be changed to get sharp and clear image of the fringe patterns. Fringe images were captured by the camera then, phase was calculated using equation (5) and unwrapped to remove $2 \pi$ discontinuities. phase error is calculated at each distance and listed in table (3). Figure (12) shows phase error of $500^{\text {th }}$ row of the unwrapped phase map associated with distances. RMS values of phase error equals to $0.0237,0.0251$ and 0.0252 rad at 500,1000, and $1500 \mathrm{~mm}$. The slight increasing in phase error with increasing the distance could be attributed increasing the noise from the surrounding area interring the camera sensor at greater distances. 


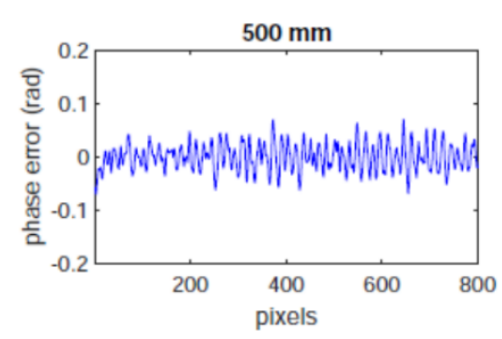

(a)

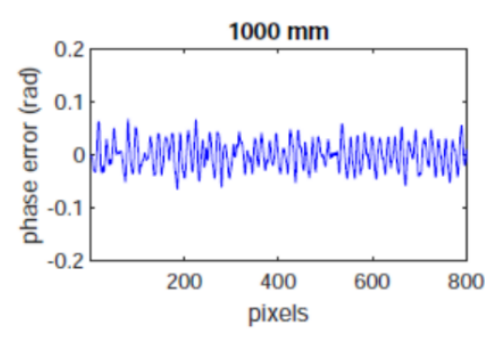

(b)

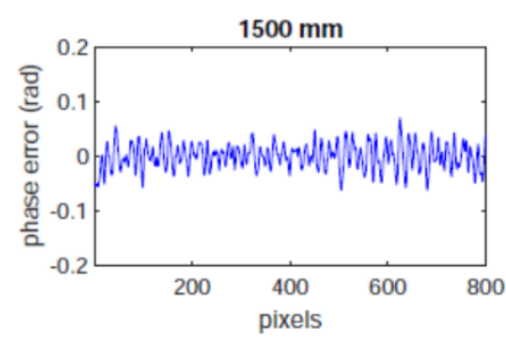

(c)

Figure (12). Phase error at distances $500 \mathrm{~mm}$ (a), $1000 \mathrm{~mm}$ (b), and $1500 \mathrm{~mm}$ (c)

To demonstrate the effect of distance on measurements, a cylindrical object is measured by projecting three sinusoidal fringe images shifted by $2 \pi / 3$ and different fringe period 35 pixels on a reference surface placed at distances 500, 1000, and $1500 \mathrm{~mm}$, Figure (13) shows one captured image of tested object at each distance. Reference unwrapped phase map is calculated at each fringe period. Again, the unwrapped phase maps are calculated with the presence of the object. The phase shift due to the object existence is calculated by subtracting the object unwrapped phase map and the reference unwrapped phase map at each distance. As the distance between the object surface and (camera and projector) increases the camera field of view increases causing the object to be represented by a smaller number of pixels in $\mathrm{x}$ and $\mathrm{y}$ direction. Also, when fringes of the same period are used at all distances the value of phase shift due to the object height decreases as the distance increases as listed in table (3). Figure (14) shows the phase shift map at each distance. As a result, the error in phase as a percentage of the measured value increases as the distance between the object surface and the system increased. This behavior appears as more irregularities in the phase shift map, so to get more accurate measurements, the object should be measured at the minimum possible distance from the camera and projector.

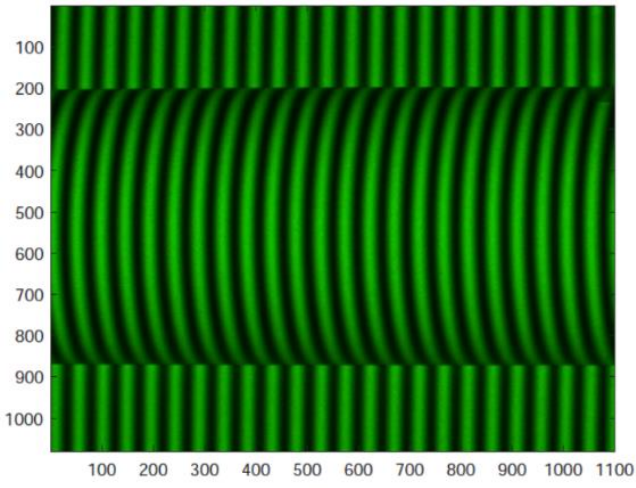

(a)

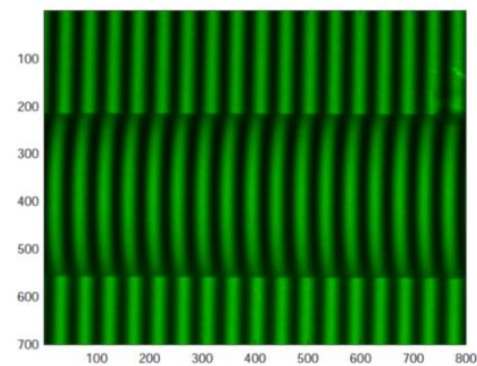

(b)

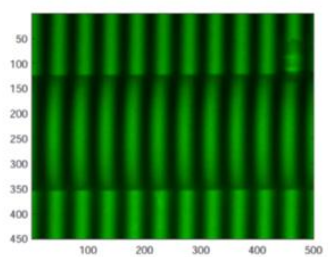

(c)

Figure (13). Captured image of test object at different distances. $500 \mathrm{~mm}(\mathrm{a}), 1000 \mathrm{~mm}$ (b), $1500 \mathrm{~mm}$ (c). 


\begin{tabular}{|c|c|c|c|}
\hline $\begin{array}{c}\text { Distance } \\
\text { Mm }\end{array}$ & $\begin{array}{c}\text { Phase error (RMS) } \\
\text { Rad }\end{array}$ & $\begin{array}{c}\text { Phase shift } \\
\text { rad }\end{array}$ & $\begin{array}{c}\text { Error } \\
\text { \% }\end{array}$ \\
\hline 500 & 0.0237 & 6.8990 & 0.343 \\
1000 & 0.0251 & 1.8287 & 1.372 \\
1500 & 0.0252 & 0.8849 & 2.847 \\
\hline
\end{tabular}

Table (3). Phase error and phase shift values at different distances.

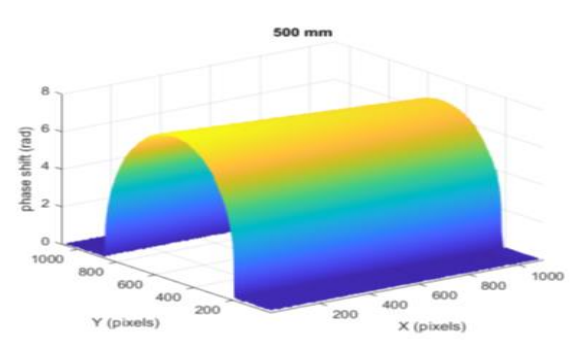

(a)

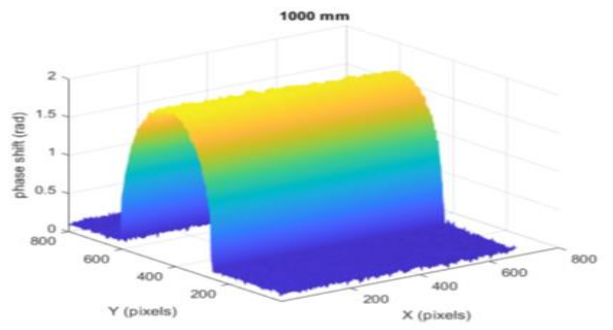

(b)

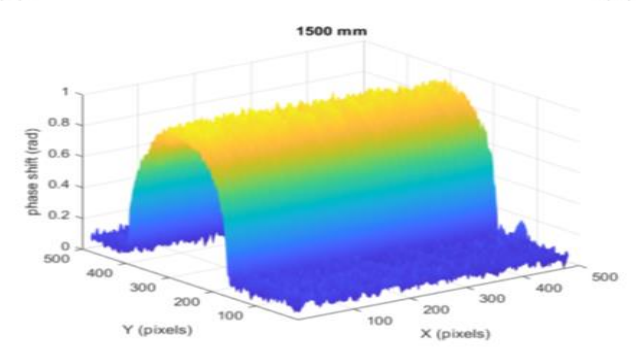

(c)

Figure (14). Phase shift maps at different distances. $500 \mathrm{~mm}$ (a), $1000 \mathrm{~mm}$ (b), and $1500 \mathrm{~mm}$ (c).

\section{3-4 Ambient illumination level.}

Fringe projection profilometry is very simple technique that could be used in different fields with varied ambient illumination level so, it is useful to study how the illumination level of the ambient affects the phase error. The system is tested at three different illumination levels (dark, median, and complete illumination) by projecting three sinusoidal fringe patterns with fringe period equals to 35 pixels and intensity amplitude (50-200) shifted by $2 \pi / 3$ on a reference surface placed $500 \mathrm{~mm}$ away from the (camera and projector). Phase map is calculated using equation (5) and unwrapped at each illumination level. Slope of $500^{\text {th }}$ row of unwrapped phase map is removed to show phase error as shown in figure (15). RMS values are calculated and listed in table (4). When the ambient illumination is changed to median level, the noise caused by the ambient light is small to cause a big change in the phase error. Whereas when the lab is completely illuminated the light source of the ambient gives higher noise level to the detected intensities and consequently, the phase measurements. As a result, the phase error value 
slightly changes from 0.0230 to $0.0243 \mathrm{rad}$ when ambient illumination is changed from dark to median level, but it shows bigger change when lab is completely illuminated reaching RMS value equals to $0.0387 \mathrm{rad}$.

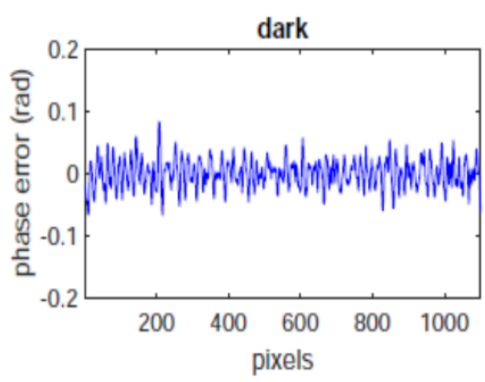

(a)

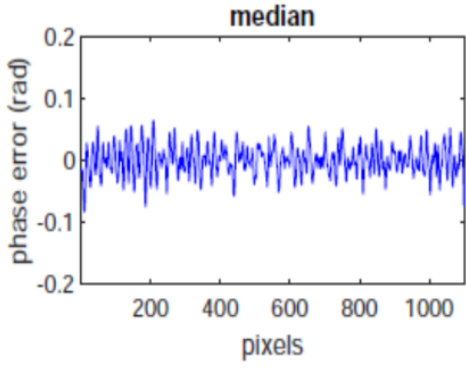

(b)

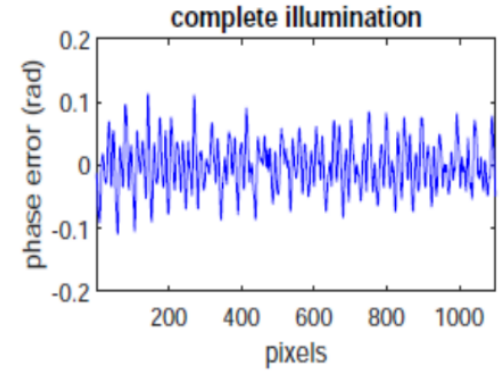

(c)

Figure (15). Phase error of $500^{\text {th }}$ row of unwrapped phase map at different ambient illumination levels. dark (a), median (b) and complete illumination (c).

\begin{tabular}{|c|c|c|c|}
\hline $\begin{array}{c}\text { Ambient illumination } \\
\text { level }\end{array}$ & $\begin{array}{c}\text { Phase error } \\
\text { (RMS) } \\
\text { Rad }\end{array}$ & $\begin{array}{c}\text { Phase shift } \\
\text { (rad) }\end{array}$ & $\begin{array}{c}\text { Error } \\
\text { \% }\end{array}$ \\
\hline Dark & 0.0230 & 6.8817 & 0.334 \\
Median & 0.0243 & 6.8775 & 0.353 \\
Completely illuminated & 0.0387 & 6.9311 & 0.558 \\
\hline
\end{tabular}

Table (4). Phase error and phase shift values at different ambient illumination levels.

As a practical example to study the effect of changing phase error with ambient illumination level, a cylindrical object is measured using three sinusoidal fringe images with fringe period equals to 35 pixels and intensity amplitude (50-200) shifted by $2 \pi / 3$. Figure (16) shows captured images of cylindrical object at different illumination level. Dark (a), median (b), and full illumination level (c). Again, phase shift due to the existence of the object is calculated as the previous section. The system parameters are kept constant so, the phase shift due to the object height is nearly constant as listed in table (4). Figure (17) shows the phase shift maps due to the object height at the three ambient illumination level. Because of increasing phase error and keeping the value of phase shift constant, the phase error as a percentage of the measured value increases from $0.334 \%$ to $0.558 \%$ of the measured value as the illumination level changed from dark to complete illumination level. 


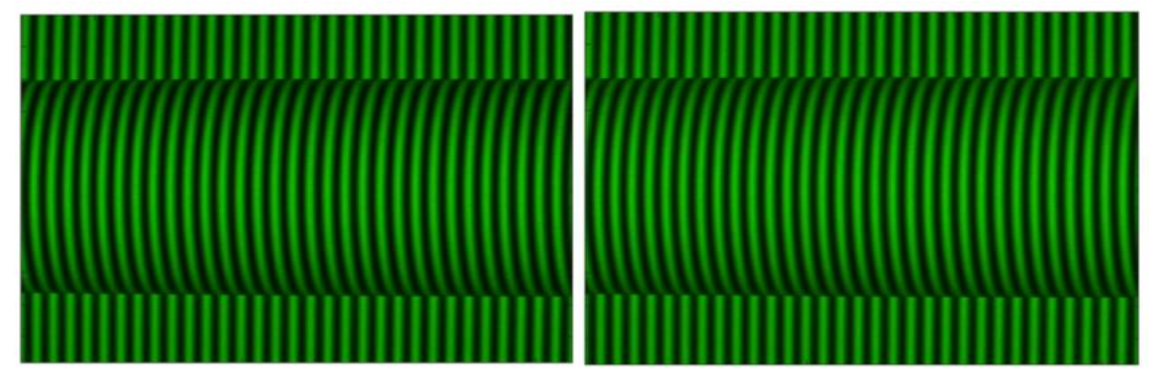

(a)

(b)

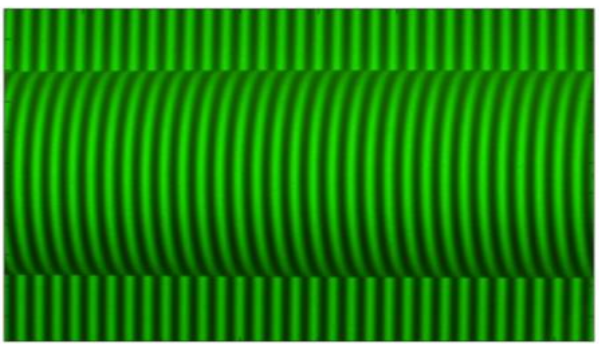

(c)

Figure (16). Captured image of cylindrical object at different illumination level. Dark (a), median (b), and full illumination level (c).

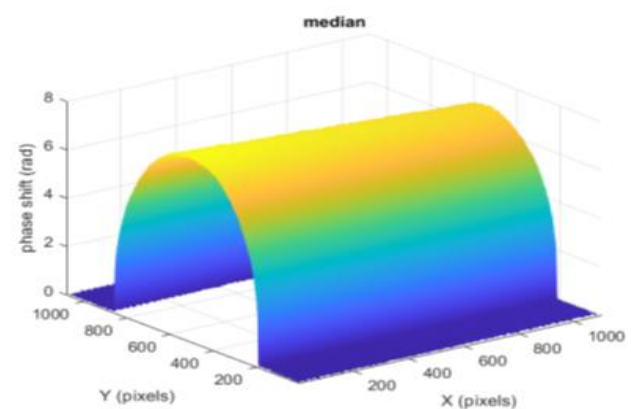

(a)

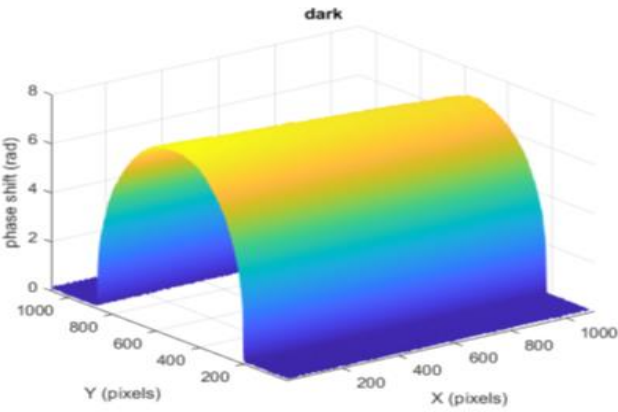

(b)

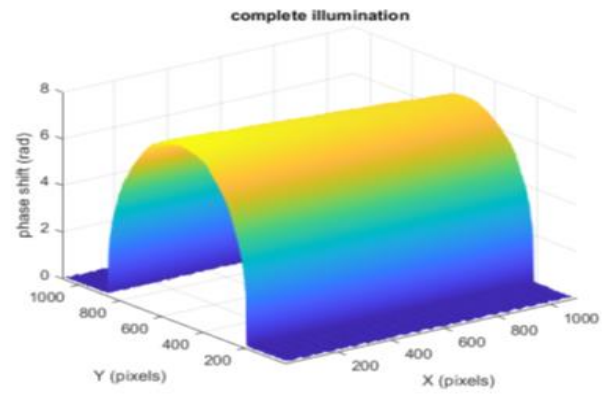

(c)

Figure (17). Phase shift at different ambient illumination levels, dark (a), median (b), and complete illumination (c). 
To judge the accuracy of the system, the system is calibrated at the optimum conditions that results minimum phase error [fringe intensity (50-220), fringe period (35 pixels), surface distance from the system $(500 \mathrm{~mm})$, and dark illumination level]. A cylindrical object is reconstructed, and 3D map is shown in figure (18). Results are given in table (5).

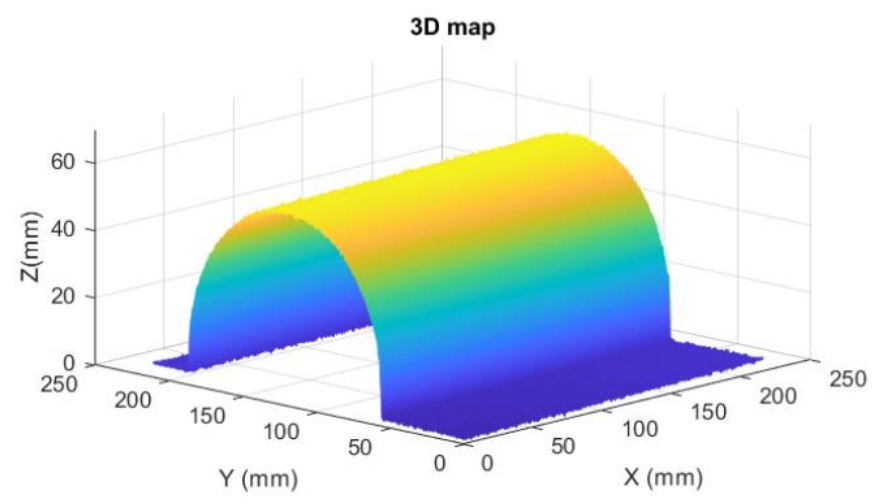

Figure (18). 3D reconstruction of the of cylindrical object.

\begin{tabular}{|c|c|c|c|}
\hline $\begin{array}{c}\text { Nominal } \\
\text { Dimension (z direction) } \\
\text { Mm }\end{array}$ & $\begin{array}{c}\text { Average Measured } \\
\text { value } \\
\text { Mm }\end{array}$ & $\begin{array}{c}\text { Error } \\
\mathrm{mm}\end{array}$ & $\begin{array}{c}\text { Standard } \\
\text { deviation } \\
\mathrm{Mm}\end{array}$ \\
\hline 56.67 & 56.55 & -0.12 & \pm 0.32 \\
\hline
\end{tabular}

Table (5). Measured value of $\mathrm{z}$ direction.

\section{Conclusion}

This paper introduces experimental investigation of the effect of system parameters fringe intensity, fringe period, surface distance from projector and camera and ambient illumination level on the phase error. The results could be summarized as following:

The system shows a linear gamma response in the range (50-220) resulting in small phase error. In the worst case, phase error does not accede 0.0387 rad without any compensation for gamma effect. this is a small value compared with other digital projecting devices such as DLP devices which gives phase error equals to 0.116 rad [17] without the compensation for nonlinear gamma response.

Phase error slightly increases from $0.0228 \mathrm{rad}$ at fringe period 25 pixels to 0.0257 rad at fringe period 40 pixels. The accuracy of system measurements will decrease with increasing the fringe period.

- $\quad$ The effect of surface distance to (camera and projector) is tested at 500, 1000, and $1500 \mathrm{~mm}$. The system shows small phase error with RMS value does not accede 0.0252 
rad. The accuracy of system measurements will decrease with increasing object surface and the (camera and projector).

- $\quad$ The system shows nearly constant small phase error 0.024 rad in dark and median level. This value increases to 0.0387 when the ambient is completely illuminated.

The system gives accurate measurements with a simple setup suitable for measuring object in controlled and uncontrolled environment.

\section{Acknowledgements}

Thanks to our colleagues in surface and engineering metrology lab., length and precision engineering division, (NIS) for their technical support.

\section{References}

1- G. Sansoni , M. Trebeschi and F. Docchio, State-of-The-Art and Applications of 3D Imaging Sensors in Industry', Cultural Heritage, Medicine, and Criminal Investigation, Sensors, 9 (2009) 568-601.

2- R. Kulkarni , P. Rastogi, Optical measurement techniques-A push for digitization, Optics and Lasers in Engineering, 87 (2016) 1-17.

3- H. Nguyen, J. Liang, Y. Wang, and Z. Wang, Accuracy assessment of fringe projection profilometry and digital image correlation techniques for three-dimensional shape measurements, Journal of Physics: Photonics, 3 (2021) 014004.

4- J. Salvi, S. Fernandez, T. Pribanic, and X. Llado, A state of the art in structured light patterns for surface profilometry, Pattern Recognition, 43 (2010) 2666-2680.

5- Xu J. and Zhang S. Status, challenges, and future perspectives of fringe projection profilometry, Optics and Lasers in Engineering, 135 (2020) 106193.

6- J. Zhang, B. Luo, X. Su, L. Li, B. Li, S. Zhang, and Y., Wang A convenient 3D reconstruction model based on parallelaxis structured light system, Optics and Lasers in Engineering, 138 (2021) 106366.

7- S. S. Gorthi and P. Rastogi, Fringe Projection Techniques: Whither we are ?,Optics and Lasers in Engineering, 48 (2) (2010) 133-140.

8- B. Li and S. Zhang, Microscopic structured light 3D profilometry: Binary defocusing technique vs. sinusoidal fringe projection, Optics and Lasers in Engineering, 96 (2017) 117-123.

9- A. Sicardi-Segade, J.C. Estrada, A. Martínez-García, and G. Garnica, On axis fringe projection: A new method for shape measurement, Optics and Lasers in Engineering 69 (2015) 29-34.

10- S. Feng, C. Zuo, L. Zhang, T. Tao, Y. Hu, W. Yin, J. Qian, and Q. Chen, Calibration of fringe projection profilometry: A comparative review, Optics and Lasers in Engineering, 143 (2021) 106622.

11- P. Yao, S. Gai, and F. Da, Coding-Net: A multi-purpose neural network for Fringe Projection Profilometry, Optics Communications, 489 (2021) 126887.

12- Y. Liu, Y. Fu, Y. Zhuan, K. Zhong and B. Guan, High dynamic range real-time 3D measurement based on Fourier transform profilometry, Optics \& Laser Technology, 138 (2021) 106833.

13- M. Zhong, F. Chen, C. Xiao, and Y. Wei, 3-D surface profilometry based on modulation measurement by applying wavelet transform method, Optics and Lasers in Engineering, 88 (2017) 243-254.

14- J. Qian, S. Feng, M. Xu, T. Tao, Y. Shang, Q. Chen, and C. Zuo, High-resolution real-time 360 3 D surface defect inspection with fringe projection profilometry, Optics and Lasers in Engineering, 137 (2021) 106382.

15- Huang J. and Wu Q. A new reconstruction method based on fringe projection of three-dimensional measuring system, Optics and Lasers in Engineering, 52 (2014) 115-122.

16- D. Mahmoud, A. Khalil, and M. Younes, A single scan longitudinal calibration technique for fringe projection profilometry, Optik, 166 (2018) 270-277.

17- S. Zhang, Comparative study on passive and active projector nonlinear gamma calibration, Applied Optics, 54 (2015) 3834.

18- P. S. Huang, C. Zhang, and F. P. Chiang, High-speed 3-D shape measurement based on digital fringe projection, Optical Engineering, 42 (2003) 163-168. 
19- X. Zhang, L. Zhu, Y. Li, and D. Tu, Generic nonsinusoidal fringe model and gamma calibration in phase measuring profilometry, Journal of the Optical Society of America, 29 (2012) 1047-1058.

20- Y. Xu, L. Ekstrand, J. Dai, and S. Zhang, Phase error compensation for three-dimensional shape measurement with projector defocusing, Applied Optics. 50 (2011) 2572-2581.

21- Z. Li, and Y. Li, Gamma-distorted fringe image modeling and accurate gamma correction for fast phase measuring profilometry, OPTICS LETTERS, 36 (2011) 154.

22- C. Zuo, S. Feng, L. Huang, T. Tao, W. Yin, and Q. Chen, Phase shifting algorithms for fringe projection profilometry: A review, Optics and Lasers in Engineering, 109 (2018) 23-59.

23- T. Huang, X. Li, X. Fu, C. Zhang, F. Duan, and J. Jiang, Arbitrary phase shifting method for fiber-optic fringe projection profilometry based on temporal sinusoidal phase modulation, Optics and Lasers in Engineering, 121 (2019) $300-306$.

24- Z. Zhao, H. Zhang, Z. Xiao, H. Du, Y. Zhuang, C. Fan, and H. Zhao, Robust 2D phase unwrapping algorithm based on the transport of intensity equation) Measurement Science and Technology, 30 (1) (2018).

25- S. Lei and S. Zhang, Digital sinusoidal fringe pattern generation: Defocusing binary patterns VS focusing sinusoidal patterns, Optics and Lasers in Engineering, 48 (2010) 561-569. 